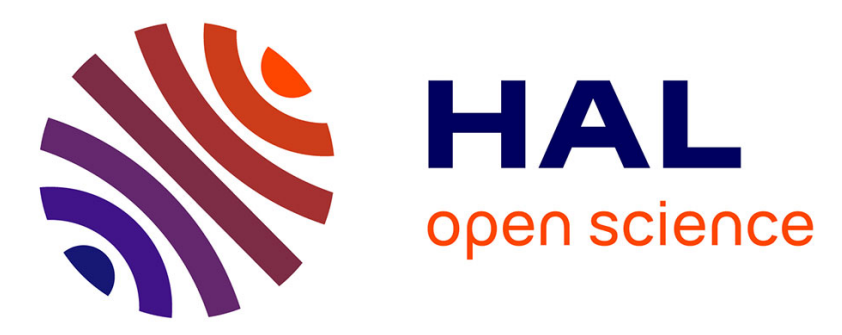

\title{
Optical and recombination properties of dislocations in cast-mono silicon from short wave infrared luminescence imaging
}

\author{
Daniel Ory, Thibaud Hildebrandt, L. Lombez
}

\section{> To cite this version:}

Daniel Ory, Thibaud Hildebrandt, L. Lombez. Optical and recombination properties of dislocations in cast-mono silicon from short wave infrared luminescence imaging. Journal of Applied Physics, 2020, 127, 10.1063/1.5140245. hal-03090687

\section{HAL Id: hal-03090687 \\ https://hal.science/hal-03090687}

Submitted on 29 Dec 2020

HAL is a multi-disciplinary open access archive for the deposit and dissemination of scientific research documents, whether they are published or not. The documents may come from teaching and research institutions in France or abroad, or from public or private research centers.
L'archive ouverte pluridisciplinaire HAL, est destinée au dépôt et à la diffusion de documents scientifiques de niveau recherche, publiés ou non, émanant des établissements d'enseignement et de recherche français ou étrangers, des laboratoires publics ou privés. 


\section{Optical and recombination properties of dislocations in cast-mono silicon from short wave infrared luminescence imaging}

Cite as: J. Appl. Phys. 127, 063102 (2020); https://doi.org/10.1063/1.5140245

Submitted: 27 November 2019 . Accepted: 26 January 2020 . Published Online: 10 February 2020

Daniel Ory, Thibaud Hildebrandt, and (D) Laurent Lombez
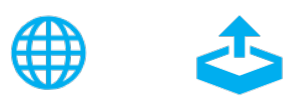

\section{ARTICLES YOU MAY BE INTERESTED IN}

Visualizing light trapping within textured silicon solar cells

Journal of Applied Physics 127, 063104 (2020); https://doi.org/10.1063/1.5131173

Advanced Thermoelectrics

Journal of Applied Physics 127, 060401 (2020); https://doi.org/10.1063/1.5144998

Enhancement of light absorption in a $\mathrm{WS}_{2}$ monolayer using spacer and Au layers

Journal of Applied Physics 127, 063101 (2020); https://doi.org/10.1063/1.5131699

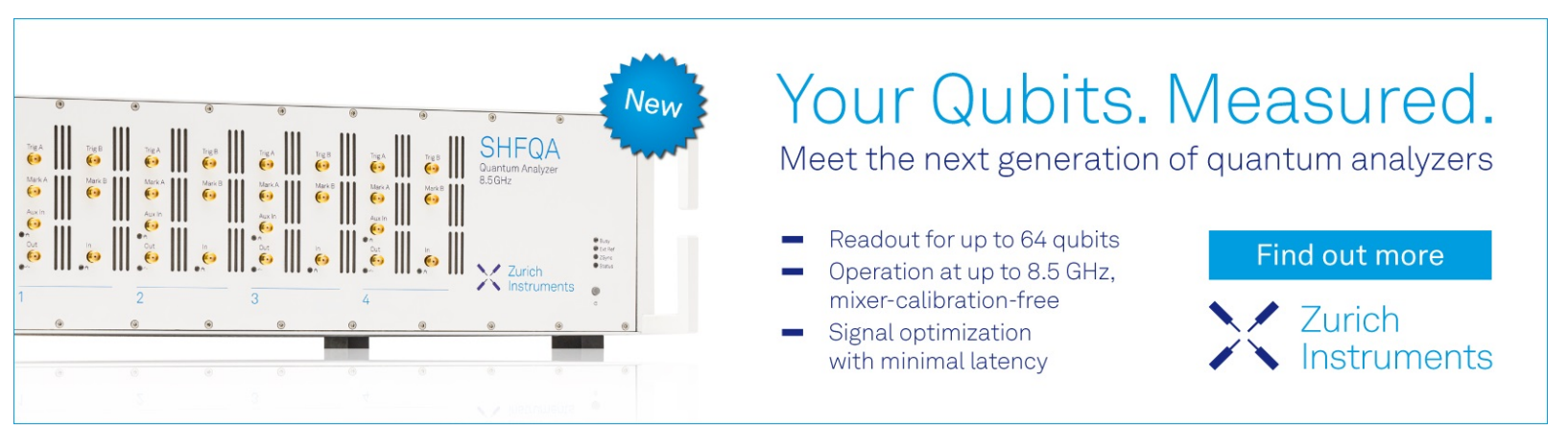




\title{
Optical and recombination properties of dislocations in cast-mono silicon from short wave infrared luminescence imaging
}

Cite as: J. Appl. Phys. 127, 063102 (2020); doi: 10.1063/1.5140245

Submitted: 27 November 2019 - Accepted: 26 January 2020 .

Published Online: 10 February 2020

Daniel Ory, ${ }^{1,2, a)}$ (iD) Thibaud Hildebrandt, ${ }^{1}$ and Laurent Lombez ${ }^{2,3}$ (D)

\author{
AFFILIATIONS \\ ${ }^{1} E D F, 18$ Boulevard Thomas Gobert, 91120 Palaiseau, France \\ ${ }^{2}$ IPVF, UMR 9006, 18 Boulevard Thomas Gobert, 91120 Palaiseau, France \\ 3INSA-CNRS-UPS, LPCNO, 135 Avenue Rangueil, 31000 Toulouse, France
}

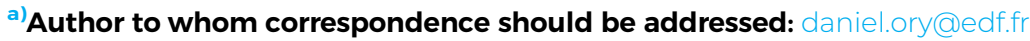

\begin{abstract}
We demonstrate the use of the D1/D2 defect luminescence spectral range ( $1400 \mathrm{~nm}<\lambda<1700 \mathrm{~nm}$ ) of dislocations in cast-mono silicon to access geometrical tilt and opto-electronic recombination properties in their vicinity. The angle between dislocation and surface was determined thanks to the spatial asymmetry of the PL intensity in the regions close to the dislocations. Our optical model relies on the classical recombination-diffusion continuity equation as well as simple optical absorption and light propagation properties. It takes advantage of the optical transparency of silicon for wavelengths above $1400 \mathrm{~nm}$. Carrier transport properties such as nonradiative lifetime around that location are also investigated. The model is discussed with a parameter study and the unicity of the extracted parameter set has been confirmed. As an example, we find out an angle of a selected dislocation pattern of about $25.4^{\circ}$ with respect to the surface and an effective lifetime of $0.8-0.9 \mu \mathrm{s}$.
\end{abstract}

Published under license by AIP Publishing. https://doi.org/10.1063/1.5140245

\section{INTRODUCTION}

Dislocations are hardly avoidable in the directional solidification system for the silicon growing process, even for the emerging quasimono (or monolike or cast-mono) technology wherein seeds obtained by the Czochralski process and free from dislocation are used to control the crystalline direction. Dislocations in silicon have been known for a long time to be detrimental to the performances of opto-electronic devices like solar cells, by affecting their bulk diffusion length ${ }^{1}$ or overall efficiency. ${ }^{2}$ They have been, therefore, studied by employing a wide range of characterization techniques and several models to describe their behavior have been proposed. ${ }^{3,4}$ The combination of chemical treatment, ${ }^{5,6}$ improved optical analysis, ${ }^{7}$ and then transmission electron microscope ${ }^{8}$ has allowed describing in detail the geometrical properties as well as the growth and the diffusion of these dislocations. Other studies described also their optical properties. In 1976 and 1977, Drozdov et al. published a first description ${ }^{9}$ of the sub-bandgap luminescence at $4.2 \mathrm{~K}$ of the dislocations in n-type as well as in p-type silicon. The different emission wavelength bands have been named
D1 (centered at $1526 \mathrm{~nm}), \mathrm{D} 2$ (centered at $1416 \mathrm{~nm}), \mathrm{D} 3$ (centered at $1327 \mathrm{~nm}$ ), and D4 (centered at $1240 \mathrm{~nm}$ ). These emissions may be observable also at room temperature and their intensities and spectral width strongly depend on the strain and temperature process applied to the material. Therefore, other luminescence signals may also appear at other wavelengths like the bands named D5 and D6. ${ }^{10}$ In addition, it has been shown extensively that the dislocations act as recombination centers for excited carriers, reducing the carrier effective lifetime. ${ }^{11,12}$ Dislocations also modify the electrical conductivity due to the modification of charge and electromagnetic field around them created by the crystal distortion. ${ }^{13}$ It may locally increase the density of impurities that are often unavoidable, such as iron. ${ }^{14}$

As the luminescence became an important way to characterize dislocation, imaging techniques have been applied in different ways for characterizing dislocations in silicon. In 1987, Guidotti et al. used scanning photoluminescence to estimate in a nondestructive way the density of dislocations on a GaAs wafer. ${ }^{15}$ Regarding silicon, Lightowlers et al. (1993) realized cathodoluminescence 
scanned images to assess the origin of D1-D2 luminescence compared to D3-D4 luminescence. ${ }^{16}$ Photoluminescence imaging of dislocations is more recent, like the work of Sugimoto et al. in 2006 about their influence on lifetime. ${ }^{17}$ Later on, Peloso showed in 2011 the link between the orientation of the dislocations by photoluminescence imaging and the polarization of their luminescence signal. ${ }^{18}$ Mankovics et al. illustrated the use of both band-to-band luminescence (BB) and D1 band luminescence images to discuss the recombination activity of the dislocations. ${ }^{19}$ Some geometrical features of the dislocation have been also investigated. Castellanos et $a l .{ }^{20}$ described the link between the eccentricity of the etch pits and the recombination strength of the dislocations. Nguyen et al. ${ }^{21}$ explored the possibility that the asymmetry of a PL profile across a subgrain boundary originates from the angle of the dislocation pattern and concluded this was not the case with their samples. In one of their recent work, ${ }^{22}$ it turned out on the contrary that the asymmetric luminescence of D1/D2 signals was associated with the inclination of the dislocations underneath the surface, which was confirmed by TEM analysis. They also noticed that the asymmetry had two preferential directions perpendicular to each other depending on the dislocation patterns. The asymmetry of D1/D2 luminescence profile of dislocations in silicon was also noticeable in the work of Tajima et al. ${ }^{23}$ even if this was not mentioned or analyzed. Only a few works exist related to the investigation of dislocation geometry by the mean of optical analysis like luminescence. For example, Nagano et al. ${ }^{24}$ or Tanuma et al. ${ }^{25}$ imaged with 2-photons excitation in three dimensions up to $200 \mu \mathrm{m}$ deep the dislocations $\mu$-PL in $4 \mathrm{H}-\mathrm{SiC}$ and reported their angle with the surface.

Our work aims at studying the optical properties of the luminescence of the dislocations in silicon, especially regarding the D1 and D2 bands above $1400 \mathrm{~nm}$ to determine the geometrical properties of the dislocation. We will show how a simple model of the D1/D2 luminescence profile extracted from a single PL image gives access to several characteristics of the dislocation: its angle with respect to the surface, the effective lifetime along the dislocation and an estimation of the surface recombination velocity. The method proposed being purely optical, it is also nondestructive for the sample. We will also present another optical method to estimate the bulk lifetime and dislocation recombination velocity from the band-to-band PL image, provided that the surface recombination velocity is known.

\section{SETUP AND DATA ACQUISITION}

Our experiment consists in recording images of the photoluminescence (PL) intensity of a silicon sample for two distinct wavelength ranges: below $1250 \mathrm{~nm}$, corresponding to the band-to-band emission, and above $1400 \mathrm{~nm}$, corresponding to the D1-D2 luminescence of the dislocations in silicon. The setup is based on a hyperspectral imager from PhotonEtc, built on an Olympus microscope, with a $2 \mathrm{~nm}$ spectral resolution and coupled to a NiRvana $640 \mathrm{ST}$ InGaAs camera cooled at $-60^{\circ} \mathrm{C}$. The excitation is realized with an $808 \mathrm{~nm}$ laser beam, spatially homogenized with a $1 \mathrm{~mm}$ diameter. The absorbed photon flux was set to $1.4 \mathrm{E} 20$ photon $/ \mathrm{cm}^{2} / \mathrm{s}$. Appropriate filters are placed into the optical path to select the desired wavelength range while eliminating the laser light.

The sample is a slab of cast-mono p-type silicon oriented [100] with resistivity about $1.2 \Omega \mathrm{cm}$ and thickness $3.12 \mathrm{~mm}$. It was mechanically and chemically polished to get a surface roughness Sa about $6 \mathrm{~nm}$. The sample surfaces were not passivated and the sample was left to ambient air during storage and experimentation. A dislocation contour whose pattern at the surface looks like a closed loop with at least one side as straight as possible has been selected and observed with an optical microscope [Fig. 1(a)], SEM [Fig. 1(b)], and the photoluminescence imaging setup [Figs. 1(c) and $1(\mathrm{~d})]$. The integrated photoluminescence images above $1400 \mathrm{~nm}$ [see Figs. 1(c) and 1(d)] depict a very asymmetrical intensity across the dislocation. This is particularly evident when plotting the PL profiles selected perpendicularly to each segment of the selected cluster. One of the sides of this cluster, indicated by the dashed yellow line in Fig. 1(c), will be further investigated. A zoom on this area is displayed in Fig. 1(d). It is to be noticed that the lateral diffusion of the minority carrier in the bulk of both sides of the dislocation contour might induce a smearing in band-to-band PL images as described and corrected in the paper of Phang et al. ${ }^{26}$ We did not correct this artifact as it is beyond the scope of this paper.

The asymmetrical shape of the PL profile might originate from the orientation of the dislocations with the sample surface. In other words, if the dislocations were contained in plane perpendicular to the surface, we would expect the PL profile to be symmetrical. The asymmetry can thus be ascribed to the tilt of the dislocation wall. It makes the PL intensity higher on the side where it is inclined. Moreover, the profile asymmetry is reversed when the luminescence is measured on the other side of the slab contrary to what was noticed by Nguyen et al. ${ }^{21}$ Also, another cause of this asymmetry could be the trace of dislocation migration during the ingot growth as described in the works of Winning et al. ${ }^{27}$ and Lawrence et al. ${ }^{28}$

To explain how the inclination of the dislocation wall makes the D1/D2 PL asymmetric, we will develop a model combining optical, geometrical, and material characteristics of the sample.

\section{MODEL}

We present here the optical model developed to describe the PL profile across the dislocation. Dislocation patterns visible in optical microscopes generally consist in closed shapes or loops formed by the juxtaposition of the intercept of the dislocations unraveled by chemical treatment with the surface of the sample (etch pits) [see Figs. 1(a) and 1(b)].

The geometry of the model is shown in Fig. 2. It considers that the luminescence is emitted along the dislocations. Those dislocations are supposed to be straight and continuous from one side of the sample to the other. We consider in-plane parallel dislocations, so the pattern on the surface forms a straight line $S L$ along the $y$-axis. We first write the model for one dislocation that is supposed to be tilted with the surface with an angle beta $(\beta)$. The growth axis (perpendicular to the surface) is set as the $z$-axis while the horizontal axis perpendicular to $S L$ is the $x$-axis [see Fig. 2(a)]. We then generalize the model by considering a juxtaposition of dislocations along $y$.

It is worth noting that the absorption coefficient of the silicon is less than $2 \mathrm{e}-7 \mathrm{~cm}^{-1}$ for wavelengths higher than $1400 \mathrm{~nm}^{29}$ Therefore, the absorption of the dislocation radiation by the silicon for these wavelengths is negligible. One can thus consider the material as transparent and develop a relatively simple optical model of the signal recorded by the camera. 
(a)

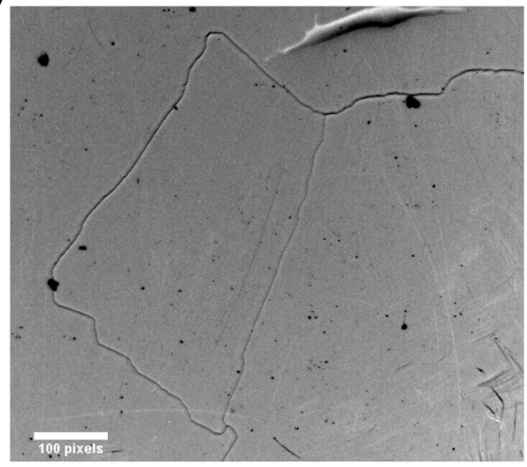

(c)

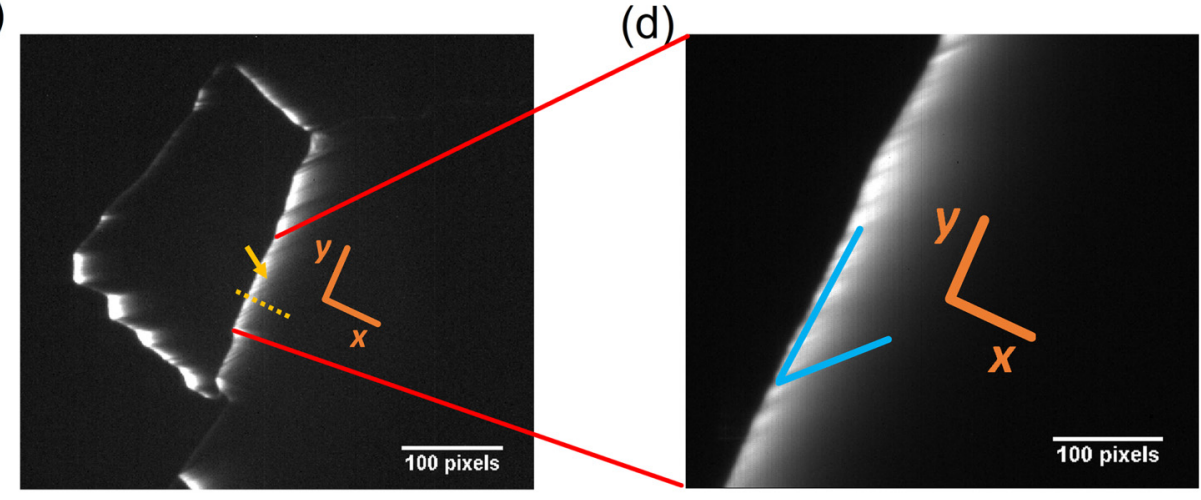

(b)

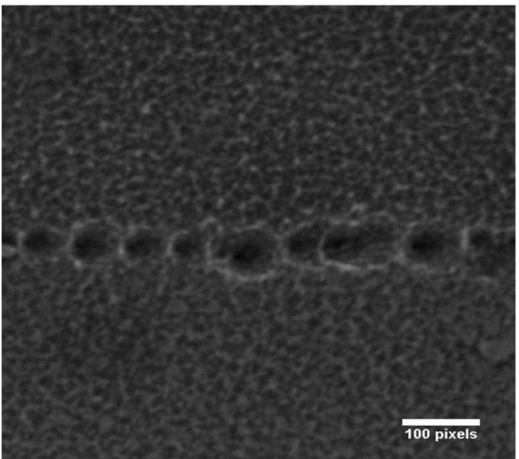

1. (a) Confocal microscope picture of the dislocation pattern (100 pixels $=323 \mu \mathrm{m})$. (b) Typical SEM image of dislocation etch pits in silicon $(100$ pixels $=100 \mathrm{~nm})$ taken from similar sample. (c) Luminescence of the dislocation pattern above $1400 \mathrm{~nm}$ ( 100 pixels $=202 \mu \mathrm{m})$. (d) Zoom out of the selected line showing the optical trace of the dislocation luminescence, with an angle of $\sim 40^{\circ}$ with the line (100 pixels $=45 \mu \mathrm{m})$.
We consider that each dislocation has an isotropic emission at any location, expressed in $\mathrm{Sr}^{-1}$. This emission is proportional to the local excess carrier density $\Delta n$. This carrier density is modeled by the Beer-Lambert law with absorption coefficient $\alpha$ associated with a $1 \mathrm{D}$ diffusion model. ${ }^{30}$ It is applied on a semiinfinite sample along direction $z$. The parameters of the diffusion model are the carrier effective lifetime $\tau$ and the front surface recombination velocity $S$ and the incident flux $\Phi$. This carrier density reads

$$
\Delta n=\frac{\Phi \alpha \tau}{\left(1-\alpha^{2} L^{2}\right)}\left(e^{-\alpha z}-\frac{\frac{S}{D}+\alpha}{\frac{S}{D}+\frac{1}{L}} e^{-\frac{z}{L}}\right)
$$

The luminescence flux impinging at the position $(x, y=0, z=0)$ generated by the radiation of the dislocation is expressed with Eq. (2), following Bouguer's law ${ }^{31}$ with distance $r$, angle $\beta$, and diffusion length $L=\sqrt{D \tau}$. The term $\delta$ accounts for the total internal reflection of the luminescence
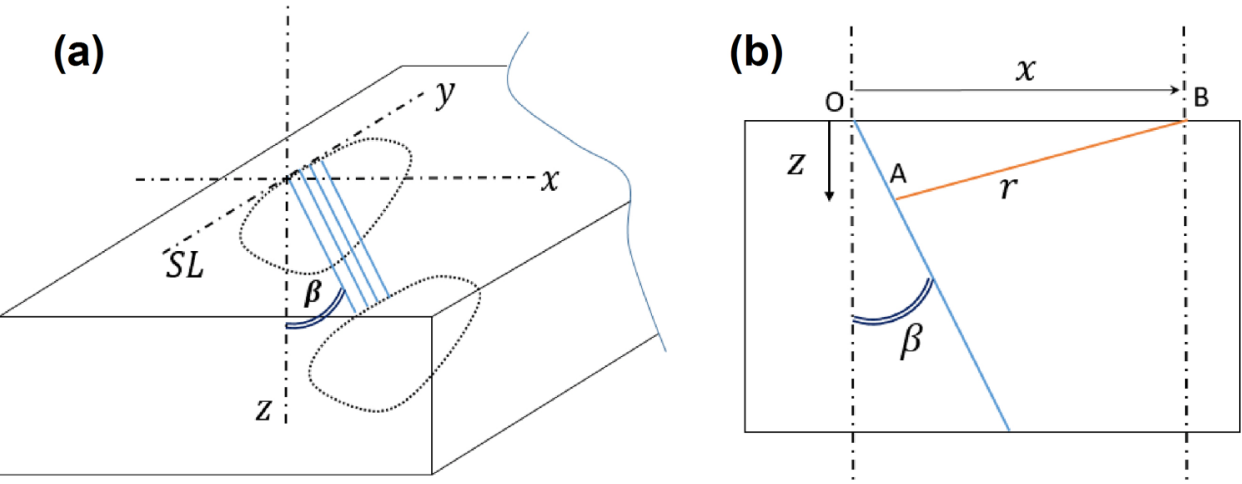

FIG. 2. (a) Schematic view of a dislocation pattern. (b) Geometrical description of one dislocation of the pattern, in the plane made by the dislocation line and the point of luminescence recorded $B$. 


$$
E(x, y, z) \propto\left[\frac{\Phi \alpha \tau}{\left(1-\alpha^{2} L^{2}\right)}\left(e^{-\alpha z}-\frac{\frac{S}{D}+\alpha}{\frac{S}{D}+\frac{1}{L}} e^{-\frac{z}{L}}\right) * \frac{z}{\left[z^{2}+(x-z \cdot \tan (\beta))^{2}+\left(\frac{y}{\rho}\right)^{2}\right]^{\frac{3}{2}}} * \delta(\gamma)\right] .
$$

With $\delta(\gamma)=\begin{array}{l}0 \text { if } \gamma>\gamma_{\text {lim }} \\ 1 \text { if } \gamma<\gamma_{\text {lim }}\end{array}$ and $\gamma_{\text {lim }}=a \sin (1 /$ (silicon refraction index $\left.)\right)$.

A linear density $\rho$ of the dislocations is assumed along $y$. One dislocation plane being arbitrarily taken at $y=0$, in which the profile is modeled and fitted, the total luminescence at the position $(x, y=0)$ is the sum of the luminescence of the $N$ dislocations distant from each other by $n=\frac{0}{\rho}$ to $n= \pm \frac{N}{\rho}$ from this origin plane, with $N / \rho$ large enough to get a converged profile.

After the convolution by the Point Spread Function (PSF) of the optical system approximated by a Gaussian kernel with parameter $\sigma$, the luminescence recorded by the camera is

$$
I_{P L}(x) \propto \sum_{n} \int_{z} E(x, z) \otimes\left[\frac{1}{\sigma \sqrt{2 \pi}} \exp \left(-\frac{x^{2}}{2 \sigma^{2}}\right)\right] d z
$$

The quantum efficiency of the camera is not included in Eq. (4) because it is rather flat between $1400 \mathrm{~nm}$ and $1600 \mathrm{~nm}$.
Moreover, the transparency of the silicon for these wavelengths means that there is no reabsorption effect.

From radiometric considerations, the luminescence emitted from the dislocation and recorded along the $x$-axis is attenuated by $1 / r^{2}$. This implies that the luminescence above $1400 \mathrm{~nm}$, incoming at $(x, y=0)$ and emitted farther than $\sim 30 \mu \mathrm{m}$ away from the surface, is negligible. We can conclude that the luminescence of the dislocations is essentially a local phenomenon despite the transparency of the silicon at those wavelengths.

To first probe the influence of the model variables, we have varied the carrier lifetime $\tau$, the angle $\beta$, the surface recombination velocity $S$, and the linear density $\rho$ of the dislocations. Figure 3 depicts the influence of each parameter on the PL profile while the other parameters are taken constant. We also show in the inset both the maximum and the average PL intensity (defined as the integral of the profile divided by the maximum intensity). (a)

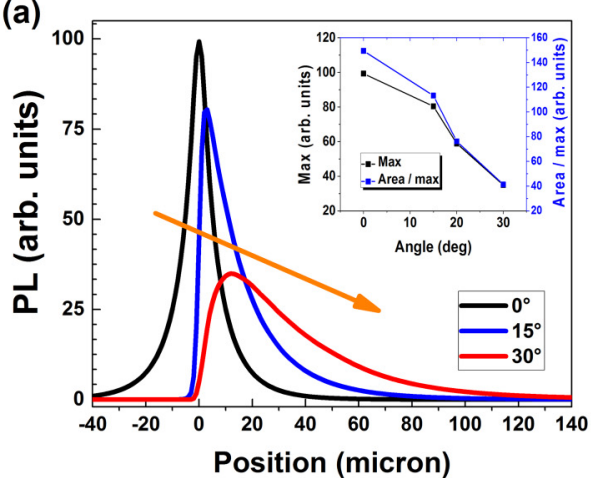

(c)

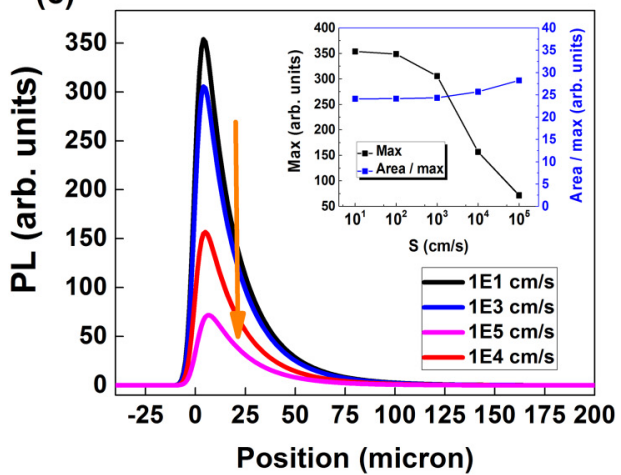

(b)

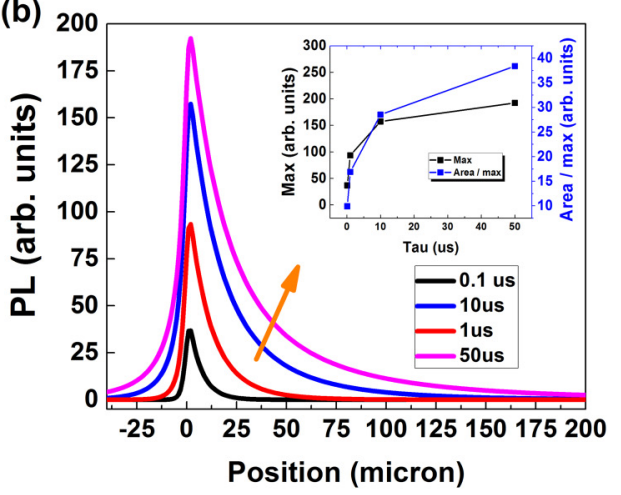

(d)

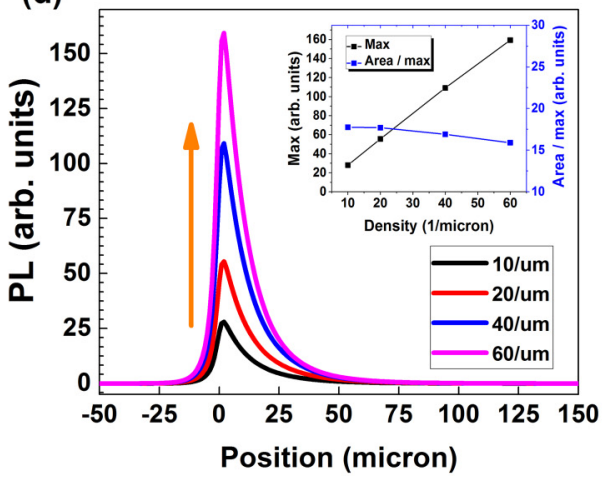

FIG. 3. Luminescence profiles calculated with the model. (a) With variable angle. (b) With variable lifetime. (c) With variable surface recombination velocity. (d) With variable linear density of dislocations. 


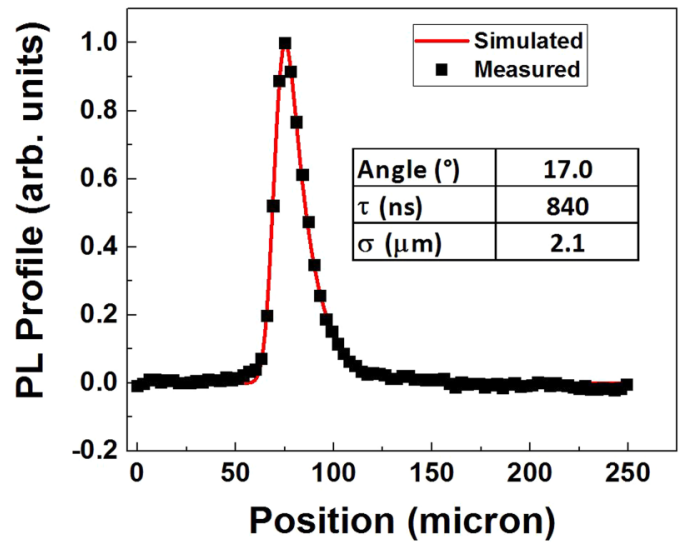

FIG. 4. Normalized luminescence profile (position shown in Fig. 1), measured and fitted.

The average value is representative of the curvature of the profile by quantifying its relative broadening.

Regarding the angle $\beta$, we expect that the profile becomes progressively asymmetrical by increasing the value of this parameter, as exhibited in Fig. 3(a). When increasing, the angle variation shifts the spatial position of the maximum profile intensity, which is no longer at the dislocation position on the surface $(x=0$ in the graphs). For example, this shift is about $3 \mu \mathrm{m}$ for $\beta=15^{\circ}$. Another important remark is that the integrated profile remains constant when the angle increases, e.g., the total number of photons recorded by the camera does not vary.

When the lifetime increases [Fig. 3(b)], the PL profile is broadened and its maximum intensity increases. This increase is more pronounced at shorter lifetime and tends to be less visible above $10 \mu \mathrm{s}$.

We also notice that the surface recombination velocity [Fig. 3(c)] influences the profile intensity and its width in a different manner than the one observe by varying the effective lifetime. The maximum intensity decreases sharply for low recombination velocities up to $1 \times 10^{4} \mathrm{~cm} / \mathrm{s}$ and then varies slowly until $1 \times 10^{5} \mathrm{~cm} / \mathrm{s}$. The relative broadening is essentially stable with a slow increase for values higher than $1000 \mathrm{~cm} / \mathrm{s}$.

The linear density of dislocations [Fig. 3(d)] increases the profile intensity while keeping the same PL profile aspect ratio. On the one hand, this allows us to use the maximum of the PL intensity as a fitting parameter to extract the linear density. On the other hand, we can fit normalized PL profiles and then ignore the linear density itself. This results in the clear advantage of avoiding the fitting of one unnecessary parameter (or experiment).

We can also deduce that the ratio of the maximum intensities of two different profiles which have similar normalized profiles corresponds to the ratio of their linear densities of dislocations, considering the surface recombination velocity being constant on the sample.

Overall, it is worth mentioning that the different parameters have different effects on the PL profile, which means that they are not cross-correlated. This ensures the unicity of the set of parameters $\{\beta, \tau, S, \rho\}$ that corresponds to one specific PL profile, provided that $S$ is larger than $1000 \mathrm{~cm} / \mathrm{s}$. Moreover, the observed variations can be used to qualitatively compare different dislocations.

Now that we have established our model and discussed the influence of the parameters, we will make use of it to fit and analyze our experimental data.

\section{FITTING RESULTS}

The fitting procedure minimizes the square of the difference between the normalized measured and modeled intensity profiles (as said before, the normalization avoids fitting the linear density of dislocations). The fitted parameters are the minority carrier effective lifetime $\tau$, the dislocation angle $\beta$, and the PSF (Point Spread Function) parameter $\sigma$. The fit of the PSF has been introduced to account for the optical response of the system and remains stable in all cases at about $2 \mu \mathrm{m}$. For the fitting procedure, we have considered a value of $1 \times 10^{5} \mathrm{~cm} / \mathrm{s}$ for the surface recombination velocity to take into account the absence of passivation layer on the surface.

As shown in Fig. 4, the model fits well the measured luminescence profile with adjusted- $\mathrm{R}^{2}$ equal to 0.997 . The parameters obtained from the fit are displayed in the inset of Fig. 4. To confirm the unicity of the solution, we have calculated the error between the best set of parameters and different sets of values around the optimal ones, including the surface recombination velocity that we have not fitted. The results are depicted in Fig. 5, where the calculated error is plotted against angle, lifetime, and surface recombination velocity. The unicity of the optimum is confirmed by the concave shape of the curves in the explored domain. The relative smooth curvature around the optimum values shows that the angle and the recombination velocity are comprised between the two sets $\left\{16^{\circ} ; 1 \times 10^{6} \mathrm{~cm} / \mathrm{s}\right\}$ and $\left\{17^{\circ} ; 1 \times 10^{5} \mathrm{~cm} / \mathrm{s}\right\}$, whereas the optimum lifetime is stable at about $850 \mathrm{~ns}$.

We have shown in this section that our model fits well our experimental data and that the dataset extracted is unique. The question is now to assess its consistency by comparison with other experimental approaches.

\section{DISCUSSION}

The angle $\beta$ of the plane made by the dislocations below the surface was found to be equal to $17.0^{\circ}$. This angle has been separately evaluated between $15^{\circ}$ and $17.5^{\circ}$ by measuring the position of the dislocation segment between both sides of the slab imaged with a confocal microscope. The accuracy of our model is noticeable from this point of view. Let us mention in addition that the work of Nguyen et al. ${ }^{22}$ exhibits a TEM image of an angled dislocation on a thin sample obtained by FIB. The angle is about $17.5^{\circ}$, which is similar to our result. The method we propose is, therefore, reliable to assess such a value using a simple PL experiment acquisition. However, the dislocation lines may be inclined within the dislocation plane (i.e., they may not be orthogonal in the $\mathrm{xOz}$ plane) (see Fig. 2). This is visible in Fig. 1(d) by zooming out on the luminescence profile. We notice the optical trace of luminescence with an angle $\delta \sim 40^{\circ}$ (blue lines) with the line in the plane of the surface. If we assume that this trace corresponds to the 

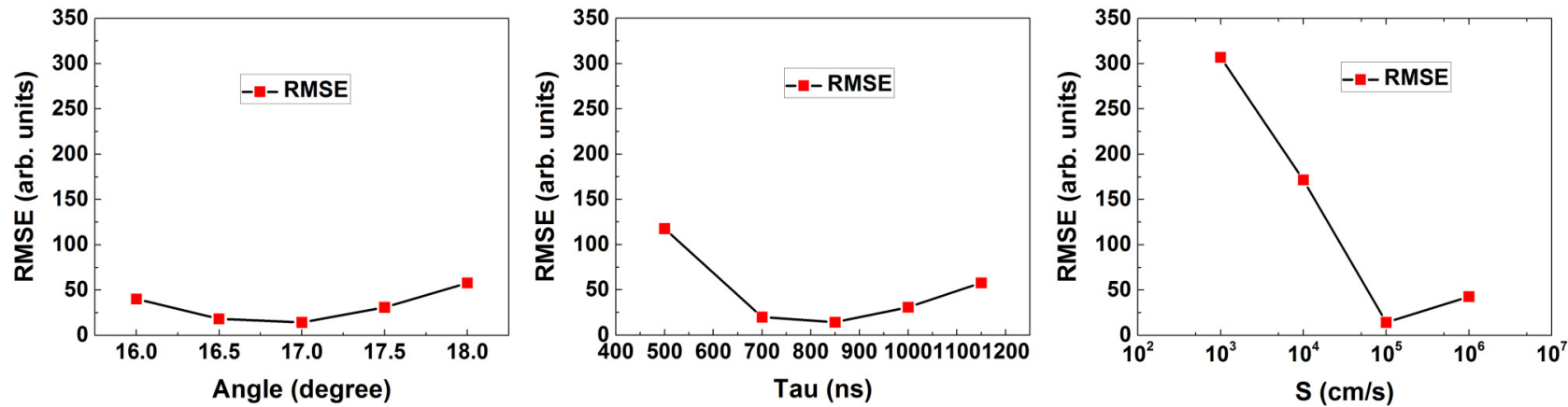

FIG. 5. Error between measured profile and theoretical one for sets of parameters varied around the optimal one. Depending on (a) angle, (b) lifetime, and (c) recombination velocity.

dislocation lines, it means that the actual angle of the dislocations with the normal of the surface is actually $\gamma \sim 25.4^{\circ}$.

Castellanos et $a l^{20}$ have also exploited the eccentricity of the etch pit to characterize recombination strength of the dislocations. The eccentricity $\varepsilon$ of an ellipse, with $a$ the major ellipse length, $b$ the minor one, and $\gamma$ the angle of the dislocation with normal to the surface reads

$$
\begin{gathered}
\varepsilon=\sqrt{1-\frac{b^{2}}{a^{2}}}, \\
\gamma=\operatorname{arcos}\left(\sqrt{1-\varepsilon^{2}}\right) .
\end{gathered}
$$

The eccentricity is 0 if the ellipse is a circle and 1 if $a$ tends to infinity, which is equivalent to $\gamma$ tending to $\pi / 2$. The assumption is that one single dislocation is a cylinder whose intercept with the surface is an ellipse depending on their angle. The eccentricity allows one to calculate the angle $\gamma$ of the dislocation with Eq. (6). Castellanos et al. reported eccentricities of about $0.4,0.45,0.6$, and 0.72 , which corresponds to angles of about $23.6^{\circ}, 26.7^{\circ}, 36.9^{\circ}$, and $46.1^{\circ}$, respectively. The value given by our experiment is, therefore, coherent with the lower range of these data.

We will now discuss the effective lifetime along the dislocation, which is influenced by the bulk lifetime and the recombination velocity of the dislocation. To do this, we have simulated the band to band luminescence of the sample, e.g., below $1250 \mathrm{~nm}$, with a simple $2 \mathrm{D}$ finite element model. The experimental image taken at this wavelength is displayed in Fig. 6(a) and the corresponding PL profile is shown in Fig. 6(b). We have then solved the differential equation of a classical diffusion-recombination model. The recombination velocity of the dislocations wall $S_{\text {disloc }}$ corresponds to one of the boundary conditions, the others being set to $1 \times 10^{5} \mathrm{~cm} / \mathrm{s}$ (top and bottom) or left not constrained. $\tau_{\text {bulk }}$ is the lifetime of the bulk as defined by the Shockley-Read-Hall model (SRH). For the sake of simplicity, the dislocation wall is considered to be perpendicular to the surface, see Fig. 6(c). We have calculated the couple $\left\{S_{\text {disloc }} ; \tau_{\text {bulk }}\right\}$ that fits the best the PL profile with two indicators: the fwhm and the contrast of the band to band PL profile across the dislocation wall (see Fig. 6). The contrast is defined as $C=\frac{I_{\max }-I_{\min }}{I_{\max }+I_{\min }}$. (a)

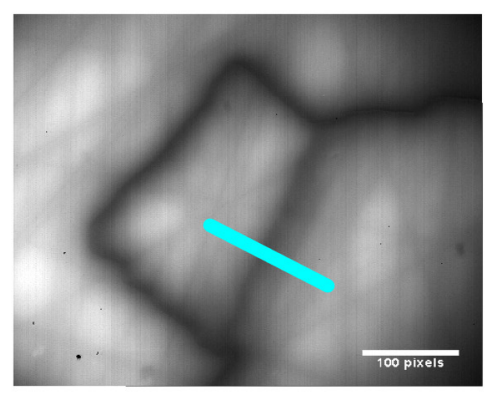

(b)

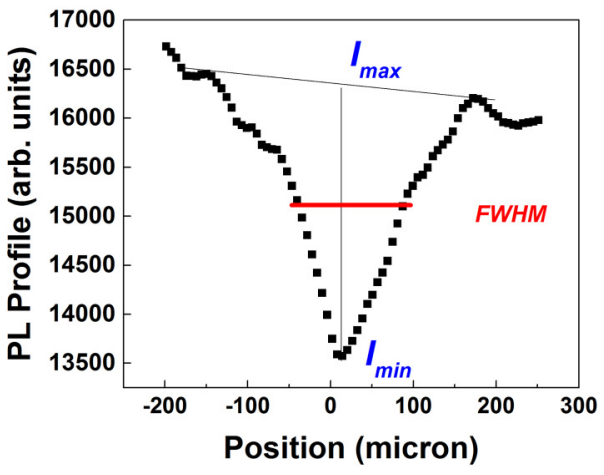

(c)

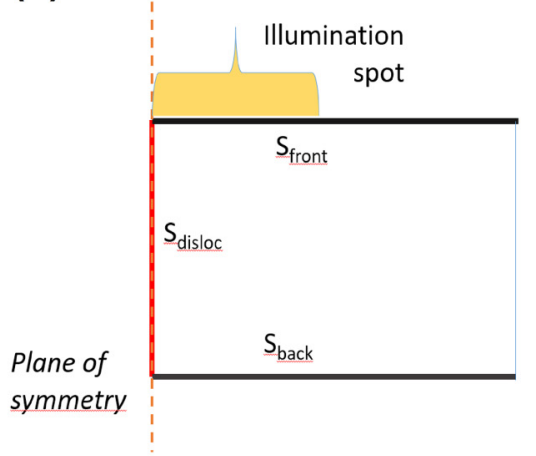

FIG. 6. (a) PL image for lambda $<1250 \mathrm{~nm}, 100$ pixels $=202 \mu \mathrm{m}$. (b) PL cross profile along blue line. (c) FEM model for vertical dislocation wall. 
The surface recombination velocity was kept constant at $\mathrm{S}_{\text {front }}=\mathrm{S}_{\text {back }}=1 \times 10^{5} \mathrm{~cm} / \mathrm{s}$. It comes out that very narrow domains of $S_{\text {disloc }}$ and $\tau_{\text {bulk }}$ fit the experimental data and we can estimate $\mathrm{S}_{\text {disloc }} \sim 2200 \mathrm{~cm} / \mathrm{s}$ and $\tau_{\text {bulk }} \sim 3.45 \mu$ s. To compare our result of $2200 \mathrm{~cm} / \mathrm{s}$ for the dislocation recombination velocity, we can refer to the work of Budhraja et al. ${ }^{4}$ They consider a value of $5000 \mathrm{~cm} / \mathrm{s}$ for fitting experimental data with their model and a value of $1000 \mathrm{~cm} / \mathrm{s}$ in some of their sensitivity studies, which is in the same range than our value. To confirm the bulk lifetime, we have passivated the surface of the sample with thin layer of a:Si-H after chemical cleaning and measured it with a modulated PL setup. ${ }^{32}$ The result is found to be about $7 \mu$ s. The discrepancy is relatively low if we consider the short lifetime of the sample. This short lifetime may be a consequence of a high concentration of iron in the sample. Indeed, the latter originates from the top of the brick (i.e., at the end of the ingot cooling) where the iron concentration is known to be high. Now, we compare this bulk lifetime value with the effective lifetime along the dislocation that has been found by fitting the D1 PL profile to be about $0.85 \mu \mathrm{s}$. To do this, we calculated the in-depth carrier density we should get if the bulk lifetime were $\tau_{\text {fit }}=0.85 \mu$ s according Eq. (1) and the one along the simulated dislocation in our FEM model with $\tau_{\text {bulk }}=3.45 \mu$ s and $S_{\text {disloc }}=2200 \mathrm{~cm} / \mathrm{s}$. The error between both is less than $15 \%$, which shows the coherence of the values obtained with our original method.

\section{CONCLUSION}

In conclusion, we have shown that by recording and fitting the luminescence profile of dislocation pattern above $1400 \mathrm{~nm}$ in cast-mono silicon wafer, one can access geometrical features of the dislocations as well as charge transport and surface recombination properties. The optical model and the experiment are relatively simple due to the long wavelength range used. We reported tilt angle of dislocations about $25.4^{\circ}$ and tilt angle of the dislocation wall of about $17^{\circ}$. Recombination velocity along the dislocations was found about $2200 \mathrm{~cm} / \mathrm{s}$ (corresponding to a linear density of 1.25 dislocation $/ \mu \mathrm{m}$ ) as well as an effective lifetime along them of about $0.8-0.9 \mu \mathrm{s}$.

\section{ACKNOWLEDGMENTS}

The authors would like to thank Alexandre Jaffré (GEEPS, CNRS, Gif sur Yvette, France) for lifetime measurement by modulated PL, Jorge Posada (EDF) for passivating the sample, Ombline Lafont (EDF), and Stefania Cacovich (CNRS) for fruitful discussions.

\section{REFERENCES}

${ }^{1}$ C. A. Dimitriadis, J. Phys. D Appl. Phys. 18, 2489 (1985).

${ }^{2}$ K. L. Pauls, K. W. Mitchell, and W. Chesarek, in Conference Record of the Twenty Third IEEE Photovoltaic Specialists Conference (IEEE, 1993), pp. 209-213. ${ }^{3}$ C. Donolato, J. Appl. Phys. 84, 2656 (1998).

${ }^{4}$ V. Budhraja, B. Sopori, N. Ravindra, and D. Misra, Prog. Photovolt. Res. Appl. 22, 1256 (2014).

${ }^{5}$ C. A. F. T. Spray, Proc. Phys. Soc. Sect. B 69, 689 (1956).

${ }^{6}$ B. L. Sopori, J. Electrochem. Soc. 131, 667 (1984).

${ }^{7}$ B. L. Sopori, Appl. Opt. 27, 4676 (1988).

${ }^{8}$ L. I. Bernewitz, B. O. Kolbesen, K. R. Mayer, and G. E. Schuh, Appl. Phys. Lett. 25, 277 (1974).

${ }^{9}$ N. A. Drozdov, A. A. Patrin, and V. D. Tkachev, Phys. Status Solidi B 83, K137 (1977).

${ }^{10}$ R. Sauer, J. Weber, J. Stolz, E. R. Weber, K.-H. Küsters, and H. Alexander, Appl. Phys. A 36, 1 (1985).

${ }^{11}$ V. Kveder, M. Kittler, and W. Schröter, Phys. Rev. B 63, 115208 (2001).

${ }^{12}$ A. Castaldini, D. Cavalcoli, A. Cavallini, and S. Pizzini, Phys. Status Solidi A 202, 889 (2005).

${ }^{13}$ G. Davies, Phys. Rep. 176, 83 (1989).

${ }^{14}$ M. Seibt, R. Khalil, V. Kveder, and W. Schröter, Appl. Phys. A 96, 235 (2009).

${ }^{15}$ D. Guidotti, H. J. Hovel, M. Albert, and J. Becker, in Review of Progress in Quantitative Nondestructive Evaluation, edited by D. O. Thompson and D. E. Chimenti (Springer US, Boston, MA, 1987), pp. 1369-1376.

${ }^{16}$ E. C. Lightowlers and V. Higgs, Phys. Status Solidi A 138, 665 (1993).

${ }^{17}$ H. Sugimoto, M. Inoue, M. Tajima, A. Ogura, and Y. Ohshita, Jpn. J. Appl. Phys. 45, L641 (2006).

${ }^{18}$ M. P. Peloso, B. Hoex, and A. G. Aberle, Appl. Phys. Lett. 98, 171914 (2011).

${ }^{19}$ D. Mankovics, R. P. Schmid, T. Arguirov, and M. Kittler, Cryst. Res. Technol. 47, 1148 (2012).

${ }^{20}$ S. Castellanos, M. Kivambe, J. Hofstetter, M. Rinio, B. Lai, and T. Buonassisi, J. Appl. Phys. 115, 183511 (2014).

${ }^{21}$ H. T. Nguyen, F. E. Rougieux, F. Wang, H. Tan, and D. Macdonald, IEEE J. Photovolt. 5, 799 (2015).

${ }^{22}$ H. T. Nguyen, M. A. Jensen, L. Li, C. Samundsett, H. C. Sio, B. Lai, T. Buonassisi, and D. Macdonald, IEEE J. Photovolt. 7, 772 (2017).

${ }^{23}$ M. Tajima, Y. Iwata, F. Okayama, H. Toyota, H. Onodera, and T. Sekiguchi, J. Appl. Phys. 111, 113523 (2012).

${ }^{24}$ M. Nagano, I. Kamata, and H. Tsuchida, Jpn. J. Appl. Phys. 52, 04 CP09 (2013).

${ }^{25}$ R. Tanuma, M. Nagano, I. Kamata, and H. Tsuchida, Appl. Phys. Express 7, $121303(2014)$

${ }^{26}$ S. P. Phang, H. C. Sio, and D. Macdonald, Appl. Phys. Lett. 103, 192112 (2013).

${ }^{27}$ M. Winning, G. Gottstein, and L. S. Shvindlerman, Acta Mater. 50, 353-363 (2002).

${ }^{28}$ J. E. Lawrence, J. Electrochem. Soc. 115, 860 (1968).

${ }^{29}$ M. A. Green and M. J. Keevers, Prog. Photovolt. Res. Appl. 3, 189 (1995).

${ }^{30}$ S. M. Sze and K. K. Ng, Physics of Semiconductor Devices, 3rd ed. (Wiley-Interscience, Hoboken, NJ, 2007).

${ }^{31}$ P. Bouguer, Essai d'optique sur la gradation de la lumière (Paris, 1729).

${ }^{32}$ R. Chouffot, A. Brezard-Oudot, J.-P. Kleider, R. Brüggemann, M. Labrune, P. Roca i Cabarrocas, and P.-J. Ribeyron, Mater. Sci. Eng. B 159-160, 186 (2009). 